haemoglobin fraction in the haemolysate may be due to its early intracellular precipitation. ${ }^{2}$ This applies to the surplus of $\alpha$-chains in $\beta$-thalassaemias, including the $\beta$-thalassaemia/ $\mathrm{Hb}$ E association ${ }^{3}$; it holds in part for the surplus of $\beta$-chains in $\alpha$-thalassaemia intermedia. " I suggest that in $\mathrm{Hb} \mathrm{E} / \alpha$-thalassaemia the expected excess of $\beta^{\mathrm{B}}$ chains precipitates intracellularly. Indeed, Dr. Wasi and his colleagues observed a small percentage of red cells with inclusion bodies, which they attributed to $\mathrm{Hb}_{4}^{\mathrm{A}}$. It is not stated whether the inclusions were preformed in vivo or had developed in vitro. It is more probable that the precipitates are composed mainly, though not of necessity exclusively, of $\beta^{\mathbb{E}}$ chains and that higher proportions of inclusions occur in red cell precursors. A low inclusion cell count in the circulation does not necessarily imply a minor degree of haemoglobin precipitation. On the contrary, it may be due to early and extensive precipitation, leading to rapid selective removal of inclusions and/or affected cells and to enrichment in cells containing $\mathrm{Hb}_{\mathfrak{s}}$, which is the most stable tetramer of this class. If $\beta^{\mathbb{B}}$ chains are less stable than $\beta^{\mathbf{A}}$ increasingly more $\beta^{\mathbf{A}}$ chains will be available to combine with the short supply of $\alpha$-chains and the $A / E$ ratio will rise. Perhaps one should expect a series of tetrameric molecules $\beta_{4}^{\mathbb{R}}, \beta_{3}^{\mathbb{R}}, \beta_{1}^{\mathbf{A}}, \beta_{2}^{\mathbb{R}}, \beta_{2}^{\mathbf{A}}, \beta_{1}^{\mathbf{R}}$, $\beta_{3}^{A}$, and $\beta_{4}^{A}$, in order of increasing stability; lability of these molecules will lead to some loss of $\beta^{\mathbf{A}}$ chains as well, so that finally only minute quanties of free $\beta^{\mathbf{A}}$ will be left over.

The suggested preferential uptake of $\alpha$-chains by $\beta^{A}$ relative to $\beta^{B}$ also may contribute to an increase of the $A / E$ ratio." However, in the relevant hybridization experiment $\beta^{A}$ chains outnumbered the $\beta^{\mathbb{E}}$ chains from the start by a factor of four. Were competition solely responsible for the high $A / E$ ratio, one would expect a large surplus of $\beta^{E}$ chains.

Finally, greater instability of $\beta^{\mathbb{E}}$ compared to $\beta^{A}$ is compatible with the fact that $\alpha$-chain deficiency combined with $\mathrm{Hb} \mathbf{E}$ is more severe, clinically and haematologically, than classical $\mathbf{H b} \mathbf{H}$ disease, although the imbalance of chain synthesis is more obvious in the latter. $-\mathrm{I} \mathrm{am}$, etc.,

Phaedon Fessas.

Aematology Section and Laboratory,

Alexandra Hospital,
Athens, Greece.

REPERBNCES

- Tuchinda, S., Rucknagel, D. L., Minnich, V., Boonyaprakob, $U$., Balankura, $\mathrm{K}$., and Suvate

V., American \}. hum. Genet., 1964, 16, 311.

- Fessas, Ph. Intern. Symposium on Comparative
Haemoglobin Structure, Thessaloniki, 1966, p.

- Fessas, Ph., Blood, 1963, 21, 21. . Pessas, Ph., and Yataghanas, X., Blood, 1968, in

the press. S., Beale, D., and Lehmann, H.
Tuchinda,
Human Geretik, 1967, 3, 312.

\section{Volvulus of the Sigmoid Colon}

SIR,-The review of 425 cases from Mulago Hospital, Kampala, Uganda, by Mr. J. J. Shepherd (3 February, p. 280) during the period 1949-65 suggests a higher incidence in Uganda than in the part of India from which I reported 40 cases during a fiveyear period in 1956.' However, to establish this I suggest it would be valuable if the actual percentage of volvulus cases to all surgical inpatients could be given, so that a basis of comparison is established. The rate at the Evangeline Booth Hospital during the years 1948-53 was about $1.5 \%$ of the surgical admissions for pelvic colon volvulus and $2.5 \%$ for all varieties of volvulus.

I recently had the opportunity in Norway of studying the rate of hospital incidence in surgical patients at the Ulleval Hospital, Oslo, for common abdominal surgical diseases from 1911 to 1960. " The "time trends " in the five diseases studied-namely, appendicitis, urolithiasis, cholecystitis and cholelithiasis, inguinal hernia, and volvulus-

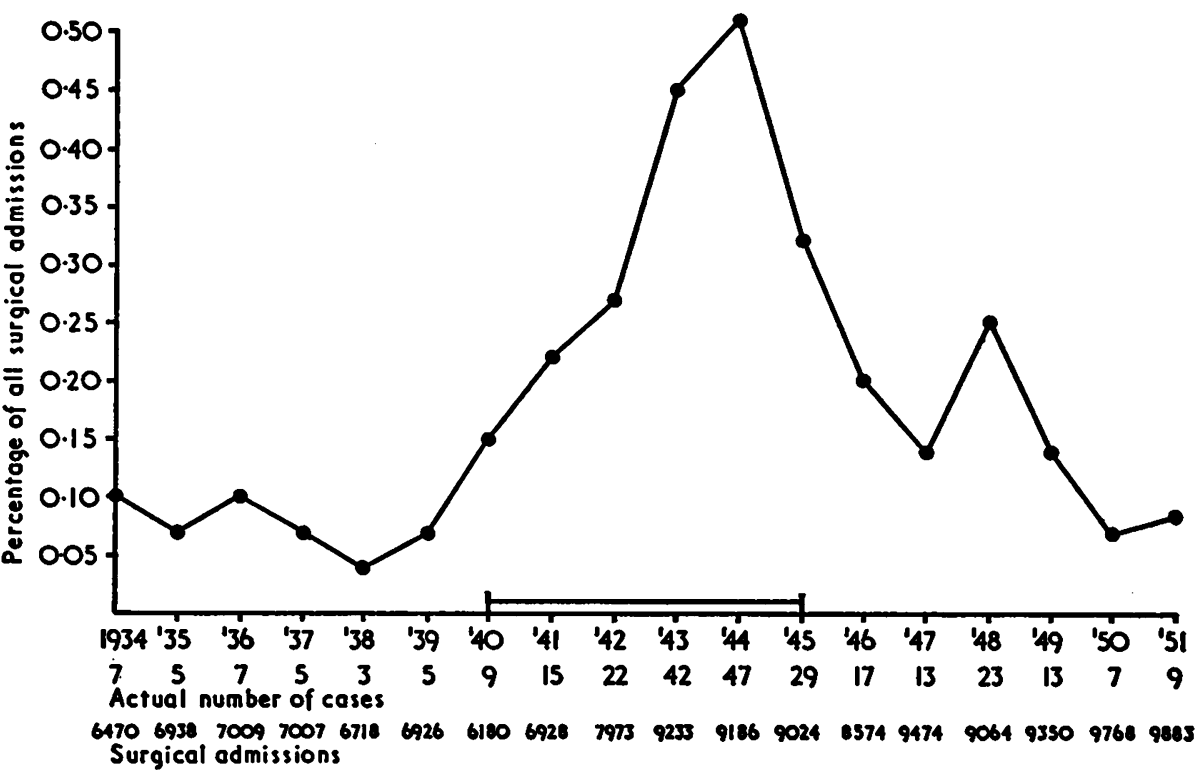

were found to be similar in pattern, although with local variations, in 10 other Norwegian hospitals from 1921 to 1960 , so far as records were available, and the pattern in appendicitis, urolithiasis, and cholelithiasis was also found to have the same "time trend " in the insured population incidence in the City of Oslo 1940-60. This seems to indicate that the hospital incidence figures can be regarded as a fairly reliable guide to the population incidence of the diseases studied when the incidence figures are obtained from a general hospital serving a wide area. The rates of incidence in the Oslo City Hospital showed a remarkably interesting relationship to both the first and the second world war years, which has already been pointed out. ${ }^{2-5}$ Volvulus was relatively common before and during the first world war, having an incidence of about $0.9 \%$ of the total surgical inpatients. It fell to under $0.1 \%$ between the two world wars, but

\section{Neurosyphilis}

SIR,-I was interested to read the paper on the changing clinical picture of neurosyphilis by Dr. R. Joffe and colleagues (27 January, p. 211). Since June 1964 I have kept a spare copy of all letters filed according to the diagnosis. During this period up until January 1968 I have seen 30 cases of neurosyphilis. The various types of disease were as follows:

Tabes dorsalis

Spinal mening $\quad \ldots \quad \ldots \quad 10$ cases Cerebral meningovascular syphilis .... 13 cases Cerebral meningosyphilis with epilepsy 2 " General paralysis of insane $\ldots . \ldots .3$

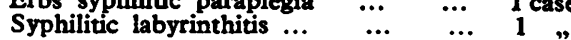

rose gradually from 1940 to reach a peak in 1944 of about $0.51 \%$ and then subsided gradually over the next six years to return to a level of about $0.09 \%$, as shown in the graph. The figures from six other hospitals in southern Norway which provided information about cases of volvulus showed a similar rise during the war, but this rise was not observed in two hospitals in northern Norway, which had a low level throughout.

These observations strengthen the sugges tion made by Bruusgaard ${ }^{3}$ that a compulsory

change of dietary habits during both world wars, from a mixed to a mainly coarser vegetarian one, had an aetiological relationship to the condition, possibly affecting a few of that proportion of the population with a congenitally long or otherwise abnormal pelvic colon. This supports the earlier observations made in India, and suggests the usefulness of further incidence studies in relation to our dietary habits and structure which could point towards remedial measures.-I am, etc.,

$$
\begin{aligned}
& \text { Institute of Urology, D. A. ANDERSEN. } \\
& \text { University of London, } \\
& \text { RBFBRENCBS }
\end{aligned}
$$

These figures are obtained from the neurological clinics of three major hospitals in the Greater London area and one in Bedfordshire, although I left Harold Wood Hospital in March 1966. Fourteen patients were attending Whipps Cross Hospital, 10 Luton and Dunstable Hospital, 4 Barnet General, and 2 Harold Wood Hospital.

The figures are comparable to those of the Newcastle series: 7 cases in a period of nine months-and the catchment population area is very similar $(900,000)$. As in the Newcastle area, several presented difficult diagnostic problems, and one patient had a familial muscular dystrophy as well as signs of a spastic paraplegia and Argyll Robertson 
pupils; and in another, a woman of 38 , the clinical picture closely resembled disseminated sclerosis. Nearly half (13 cases) were over the age of 60 , and 6 of them were over the age of 70 . It came as a surprise to find such a large number in my files. Comparable figures in the same period of other neurological diseases are subacute combined degeneration 32 cases, syringomyelia 27 cases. The figures confirm the view that the era of neurosyphilis is still very much with us.I am, etc.,

London W.1.

\section{K. W. G. HeathField.}

\section{Gastrectomy and Vagotomy}

SIR,-We read with interest the article by Mr. Alan G. Cox (3 February, p. 88), comparing symptoms after vagotomy and partial gastrectomy.

Perhaps it should be made clear that in the partial gastrectomy cases referred to the anastomosis has been made by bringing up a jejunal loop to reach the gastric stump, either in front of or behind the colon. We have shown that if this technique is avoided and a no loop gastrectomy is made those sequelae in Mr. Cox's list which cause the real distress are virtually eliminated. The loss of pyloric function and control in this as in most operations for duodenal ulcer entails the need for meals to be taken slowly, reducing the sweet course, and taking drinks at other times, but this is not important: the crux of the matter is that those sequelae such as bile vomiting, recurrent ulcer, and insidious obstructive symptoms which make up the "gastric cripple" are avoided by the no loop technique.

The best surgical procedure for the intractable duodenal ulcer will remain debatable for many years, but it is misleading to compare gastrectomy without qualifications-loop or no loop-with other methods such as vagotomy. -We are, etc.,

\section{London Hospital, \\ Douglas EAdir.}

\section{REFBRENCB} 'Taylor, H., and Eadie, D. G. A., Brit. med. J.,
1967, 1, 15.

\section{Aldosterone and Electrolyte Movements in the Colon}

SIR,-May I reply to the letter of Dr. Oliver Wrong (10 February, p. 379) written in response to our paper on the altered colonic absorption and secretion of water and electrolytes in a patient with primary aldosteronism (13 January, p. 93) ?

Firstly, it is not very surprising that in our patient sodium absorption by the colon was within normal limits. After all, sodium retention by the kidney is not a feature of primary aldosteronism, and, as we discussed in our paper, in this disease the colon may "escape" in a similar manner to the kidney from the sodium-retaining activity of aldosterone. Secondly, I do not think that Dr. Wrong is quite fair either to us or to Levitan and his colleagues ${ }^{2}$ by implying that grossly dissimilar results can be obtained by small differences in experimental technique. The composition of the solution perfused through the colon, the difference between our study and those of Levitan et al., is critical, and the choice of perfusion solution requires considerable care. Thus Levitan' admitted that he was unable to evaluate the effect of 9- $\alpha$-fluorohydrocortisone on potassium handling by the colon because the solution which he had used did not contain potassium.

Although I must congratulate Dr. Wrong and his colleagues for the excellent demonstration of the action of aldosterone on electrolyte handling by defunctioned colon, distal to a transverse colostomy, I cannot agree that this type of patient should be preferred for studies of colonic absorption. Firstly, to have normal colonic mucosa distal to a transverse colostomy is very rare. Surely colon affected by diverticulitis to such an extent that a colostomy was required cannot be regarded as normal ? Obviously it would be rarer still to encounter a patient with a colostomy and primary aldosteronism. Moreover, it would be impossible to study patients with ulcerative colitis ${ }^{4}$ or a villous papilloma, in whom colostomy is often unwise or unnecessary. Finally, by using patients with a transverse colostomy only the left side of the colon can be studied. We are more interested in the absorptive capability of the entire colon, especially because the right colon has a greater capacity for absorption than the left. ${ }^{.}$For these reasons I feel that we shall have to continue with what Dr. Wrong regards as more complicated experiments.- I am, etc.,

\section{Surgical Unit, Royal Infirmary,
Cardiff. \\ R. SHIELDS.}

\section{REPERENCES}

Levitan, R., and Ingelfinger, F. J., J. clin. Invest., 1965, 44, 801

2 Levitan, R., 尹. Lab. clin. Med., 1967, 69, 558 .

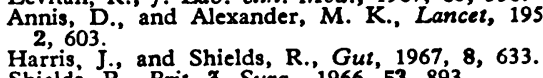
5 Shields. R.,. Brit. 7. Surg., 1966, 53, 893.

Levitan, R., Fordtran, J. S., Burrows, B. A., and
Ingelfinger, F. J., f. clin. Invest., 1962, 41, 1754 .

\section{Haemophilus Epiglottitis}

SIR,-Dr. L. Crome and others (24 February, p. 508) state, "In spite of stridor and croup in our two cases the fatal outcome was not due to upper respiratory obstruction, and intubation was not helpful. Death was probably caused by pulmonary collapse, peripheral circulatory failure, and rapidly developing cerebral oedema."

The three pathological findings to which they refer are all the end result of other processes, and can be explained by the presence of respiratory obstruction. Hypoxia and hypercarbia are well-known causes of cerebral oedema; respiratory and/or metabolic acidosis will cause "peripheral circulatory failure" and could explain the failure of intubation alone to produce clinical improvement; and respiratory obstruction will cause pulmonary collapse.

It is difficult to understand how they can consider that upper respiratory obstruction did not cause death (in the sense that it inexorably led up to the final collapse) when one child had stridor and was dyspnoeic and cyanosed, and the other had stridor, tachypnoea, and tachycardia.
Unless Dr. Crome and his colleagues can produce evidence of a normal acid-base state, and show that cerebral oedema was not due to hypercarbia and/or hypoxia, I do not think that their implication that Haemophilus influenzae infection of the epiglottis causes death other than from the sequelae of its local effect is valid. If they have drawn attention away from the importance of relief of respiratory obstruction, the correction of hypoxia and hypercarbia, and the treatment of metabolic acidosis and cerebral oedema, if present, they have not helped the management of this difficult disease.-I am, etc.

Royal Aberdeen Hospital
for Sick Children,

H. G. Pledger. for Sick
Aberdeen.

SIR,-If endotracheal intubation was " not helpful " in the fatal cases of haemophilus epiglottitis described by Dr. L. Crome and others (24 February, p. 508) might this have been because it was left too late?

Early tracheal intubation in infants with respiratory distress bypasses any upper respiratory obstruction, facilitates aspiration of exudate and secretions, reduces dead space, and enables intermittent positive pressure respiration to be carried out; once the child is adequately oxygenated elective tracheostomy can be performed. Because resistance to gas flow in the respiratory passages varies inversely as the fourth power of the diameter, the smallest degree of airway obstruction in a child can produce an intolerable increase in airway resistance. Thus if stridor occurs with signs of hypoxia (cyanosis, tachycardia, restlessness) tracheal intubation is needed urgently.

Dr. Crome concludes that death was not caused by upper respiratory obstruction but was probably due to pulmonary collapse, peripheral circulatory failure, and cerebral oedema. If oxygen therapy is given without relieving airway obstruction absorption collapse of the lung becomes very likely ; and hypoxia is a very potent cause of circulatory failure and cerebral oedema.-I am, etc.,

Epping, Essex.

J. D. HILL.

\section{Deaths from Asthma}

SIR, - I would like to make a few points on the management of acute and chronic bronchial asthma. You state in your leading article (10 February, p. 329) that practitioners should give patients in status asthmaticus intravenous or intramuscular hydrocortisone every 15 minutes. Oral prednisolone given in a dose of $20 \mathrm{mg}$. before the patient is sent to hospital, where he can be given a further $20 \mathrm{mg}$., will probably be of greater value. This is because the blood levels produced by parenteral hydrocortisone do not last very long, especially when given intravenously. The bronchodilator effect of corticosteroids takes from six to 10 hours to become established. Oral prednisolone is well absorbed and maintains adequate blood levels till this effect is produced. We usually find $40 \mathrm{mg}$. a day of prednisolone an adequate dose. The arterial $\mathrm{Po}_{2}$ of patients in status 